\title{
La société chinoise, la plus maseuline du monde
}

\section{En espérant un fils..., la mascultinisation de la population chinoise (Isabelle Attané)}

Dominique Labie

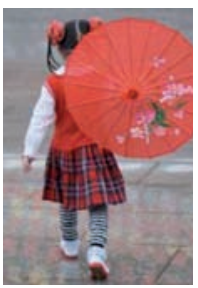

\author{
D. Labie : Inserm U567, CNRS UMR 8104, \\ Institut Cochin, \\ 24, rue du Faubourg Saint-Jacques, \\ 75014 Paris, France. \\ dominique.labie@inserm.fr
}

Des bouleversements profonds ont marqué la Chine, politiques, sociologiques, démographiques. Avec un développement économique considérable, la Chine est aujourd'hui une société très inégalitaire. La transition démographique se traduit par une population comportant peu de jeunes et dont le taux de masculinité est le plus élevé du monde; le déficit en femmes y est de l'ordre de 60 millions. Un livre d'Isabelle Attané, $\varepsilon n$ espérant un fils..., publié aux Éditions de l'Ined, analyse le processus [1]. L'auteur y recense les données démographiques factuelles, puis analyse l'ensemble de normes et de valeurs, traditionnelles et socioéconomiques, qui ont favorisé ce phénomène.

Cette évolution est atypique, les données de natalité et de mortalité montrant habituellement, dans une population fermée, un léger excès en faveur des femmes, par surmortalité masculine. Commune à de nombreux pays asiatiques (Inde, Corée de Sud, Taiwan, etc.), elle atteint aujourd'hui des proportions inédites en Chine, marquée par un déficit de filles à la naissance et par une surmortalité féminine au cours de l'enfance qui se prolonge tout au long de l'existence.

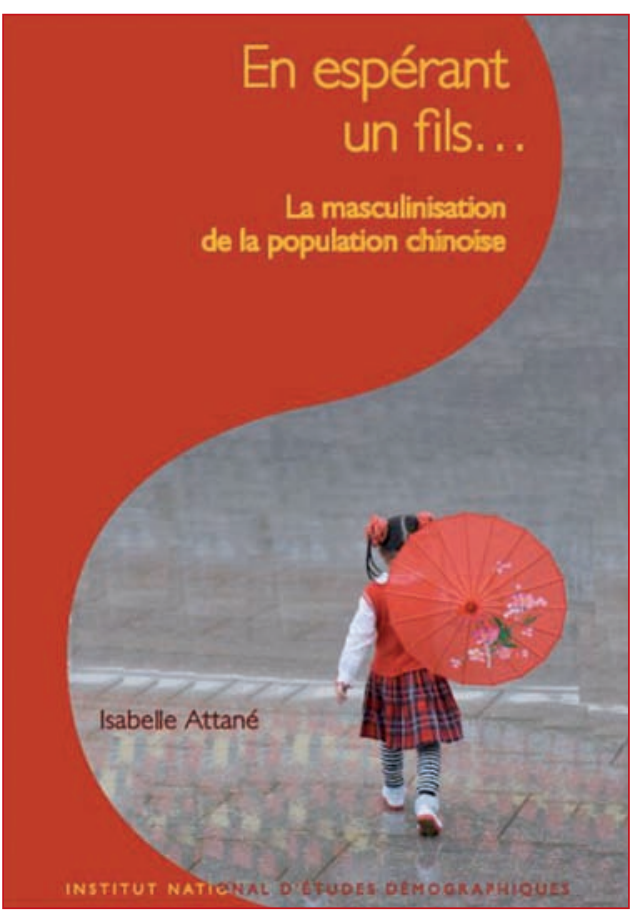

La Chine est traditionnellement marquée par une masculinité et une mortalité des femmes (signalées par les jésuites au XVII ${ }^{e}$ siècle), et l'élimination des filles, qui était une pratique ancestrale, a connu une augmentation considérable. Le déficit de filles entre deux recensements (1989-2000) a été de 11 millions, favorisé par les techniques modernes d'échographie, facilement accessibles et peu onéreuses. Leur usage pour la détermination du sexe, interdit par la loi, est peu contrôlé, et la corruption répandue. La mortalité infantile touche également surtout les filles, et ce dès les premiers jours de l'existence, avec en moyenne 112500 décès par an, par noyade ou étouffement de l'enfant, et se poursuit jusqu'à 5 ans. Ce phénomène est 
plus massif dans les campagnes qu'en ville et variable d'une province à l'autre et selon les groupes ethniques. II y avait, en 2005, 118 garçons pour 100 filles. Au cours de la vie le régime de mortalité reste défavorable aux femmes dans la vie quotidienne et au cours d'accouchements en dehors du milieu hospitalier. Ce déséquilibre d'ensemble est de toute évidence un choix des familles; lié à une appartenance religieuse, il est accepté par le confucianisme, et l'est beaucoup moins chez des populations musulmanes ou chrétiennes. II est aussi en relation avec l'extrême pauvreté, le garçon est perçu comme une force de travail et un recours pour la vieillesse.

L'auteur insiste sur un statut patriarcal confucéen qui a toujours marqué la société chinoise traditionnelle et qui imprègne les mentalités. La tradition du mariage patrilocal est profondément enracinée; l'épouse quittant sa propre famille biologique pour être entièrement soumise à sa belle-famille, la petite fille n'est pas un investissement rentable. Seul le fils pourra assurer la lignée familiale et le culte des ancêtres, la femme étant avant tout l'instrument de la fécondité. Cependant, on a vu lentement se dessiner un mouvement féministe. Le mouvement Taiping au XIXe siècle condamnait bigamie, prostitution, adultère et pieds bandés. Le régime communiste, à partir de 1949, a voulu l'émancipation des femmes, proclamé leur égalité et une loi sur le mariage. La réforme agraire, en collectivisant le travail, a mobilisé les femmes comme les hommes, mais au cours des années 1980, une politique de redistribution de lopins de terre a favorisé la reconstitution du noyau familial. Le gouvernement a lancé dans plusieurs provinces un programme intitulé «Chérir les filles», accordant aux familles qui n'ont que des filles des avantages économiques.

Dans une société très inégalitaire, les disparités socioéconomiques favorisent la masculinisation. La scolarisation est souvent discriminatoire avec, en conséquence, une moindre compétitivité des filles face à l'emploi. Tous ces facteurs jouent dans la forte attente des familles vis-à-vis d'un fils. Depuis 1990, divers travaux, dont ceux de l'Ined, mais aussi les statistiques chinoises, ont étudié l'incidence factuelle des pratiques familiales et constaté que des millions de filles n'ont jamais été déclarées à leur naissance; il est plus facile de faire disparaître un enfant non déclaré et qui n'a donc «jamais existé ». On est autorisé à en concevoir un autre en évitant la pénalité imposée en cas de second enfant par la loi chinoise sur l'enfant unique. Toutefois, l'infanticide des filles serait circonscrit à des villages isolés, et l'abandon n'est qu'un dernier recours, la pratique majeure restant l'avortement sélectif, choix économique plus que choix moral pour le couple, auquel s'ajoute l'inégalité d'accès aux soins. Les variables à l'origine de ces pratiques discriminatoires sont culturelles et socio-économiques. L'auteur appelle fécondité «politique », les moyens employés pour résister aux mesures de contrôle. Toutes ces variables rendent l'interprétation des faits complexe. Une tentative d'explication retient, avec la tradition du mariage patrilocal, quatre variables essentielles: l'extrême pauvreté, le niveau de la fécondité, le niveau d'éducation des hommes et le niveau de solidarité avec la génération des grands-parents. Avec la politique de l'enfant unique qui veut que la conception d'enfants de rang suivant $(2,3 \ldots)$ soit autorisée si le premier est décédé, tout est réuni pour une masculinisation massive de la société chinoise et une transition démographique atypique.

Ce phénomène de la Chine actuelle n'est pas unique au monde, mais son intensité et l'importance de la Chine en font une donnée économique et sociale nouvelle. On constate déjà un déséquilibre des sexes sur l'offre matrimoniale, pouvant aboutir mécaniquement à une diminution de la natalité. Il entraîne un retard généralisé du mariage des hommes avec des épouses de plus en plus jeunes (la loi n'autorise en principe le mariage des filles qu'à partir de 20 ans), mais aussi avec des veuves et des divorcées. Certains frontaliers épousent des Vietnamiennes. La croissance démographique elle-même pourrait-elle subir les conséquences de ce déséquilibre? On peut prévoir, entre 2030 et 2050 , que $20 \%$ des hommes (1,5 million chaque année) seraient dans l'impossibilité de se marier, avec les conséquences sociales d'une lignée éteinte, d'une vieillesse non assurée. Quel pourrait en être l'impact sur le statut des femmes? À l'heure actuelle, les violations des droits fondamentaux sont fréquentes: enlèvements, viols, mariages forcés, une certaine marchandisation des femmes. On peut, à l'inverse, trouver à cette masculinisation des aspects positifs en faveur des femmes: valorisation de leur force de travail, possibilité pour elles d'un éventail de choix professionnels et matrimoniaux. Il est pour l'instant impossible de savoir si les pratiques discriminatoires, cause et conséquence de la masculinisation de la société, représentent une évolution durable ou un phénomène transitoire. On ne peut ignorer qu'en Chine, pays le plus peuplé et le plus masculin du monde, 600000 filles sont chaque année éliminées par la conjonction de pratiques ancestrales, d'inégalités socioéconomiques profondes et de lois restrictives de contrôle des naissances. $\diamond$

Chinese society, the most masculine of the world

\section{CONFLIT D'INTÉRÊTS}

L'auteur déclare n'avoir aucun conflit d'intérêts concernant les données publiées dans cet article.

\section{RÉFÉRENCE}

1. Attané I. En espérant un fils..., La masculinisation de la population chinoise. Paris : Ined, $2010: 240$ p.
TIRÉS À PART

D. Labie 

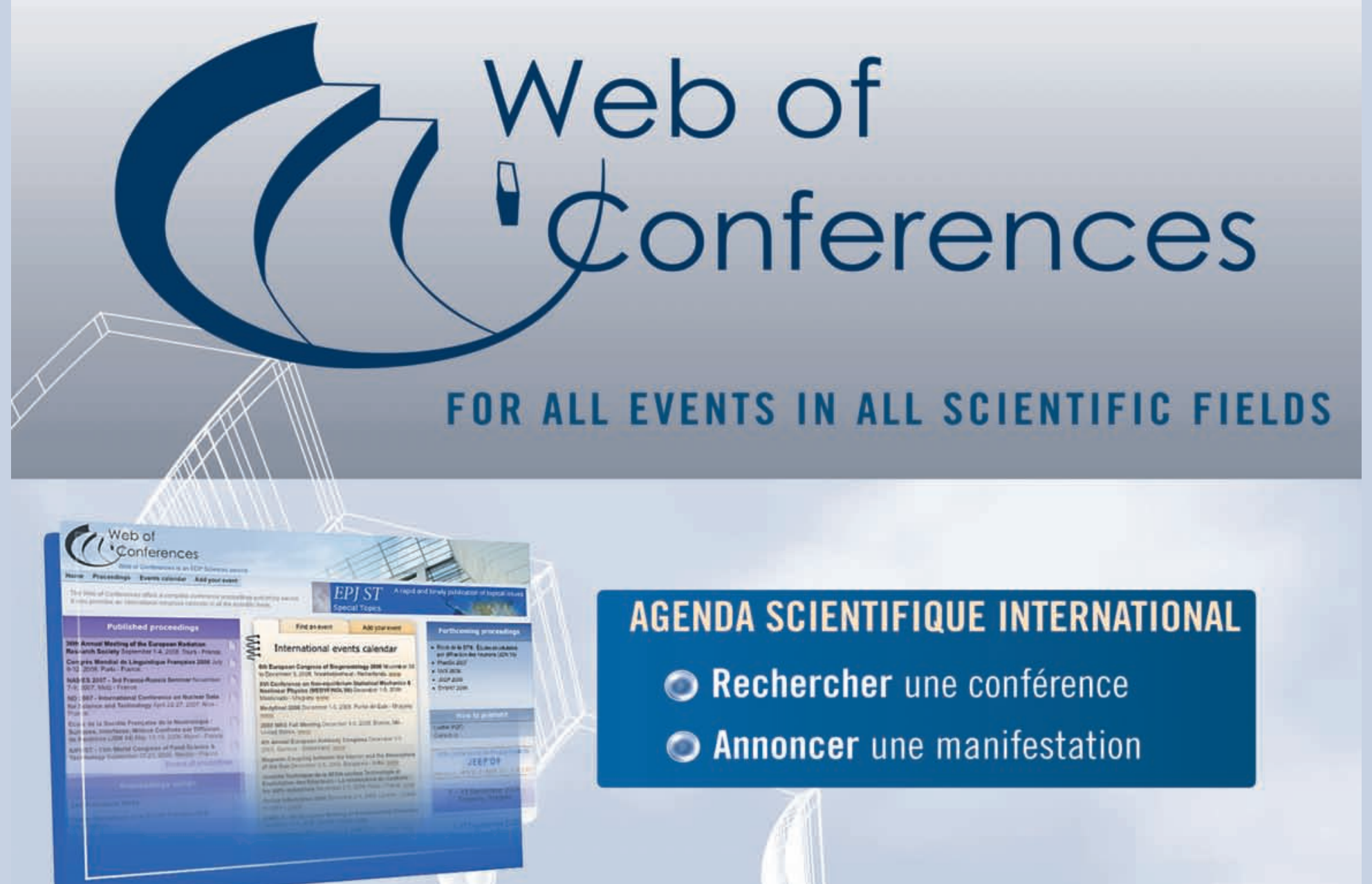

\section{PUBLICATION D'ACTES DE CONFERENCE}

- Publication en ligne sur un site

- Diffusion en open-access web dédié à la conférence

- Archivage et maintenance

- Articles parfaitement identifiables et citables (DOI, Crossref)

- Création de collections

Annoncez gratuitement vos prochaines manifestations sur www.webofconferences.org 

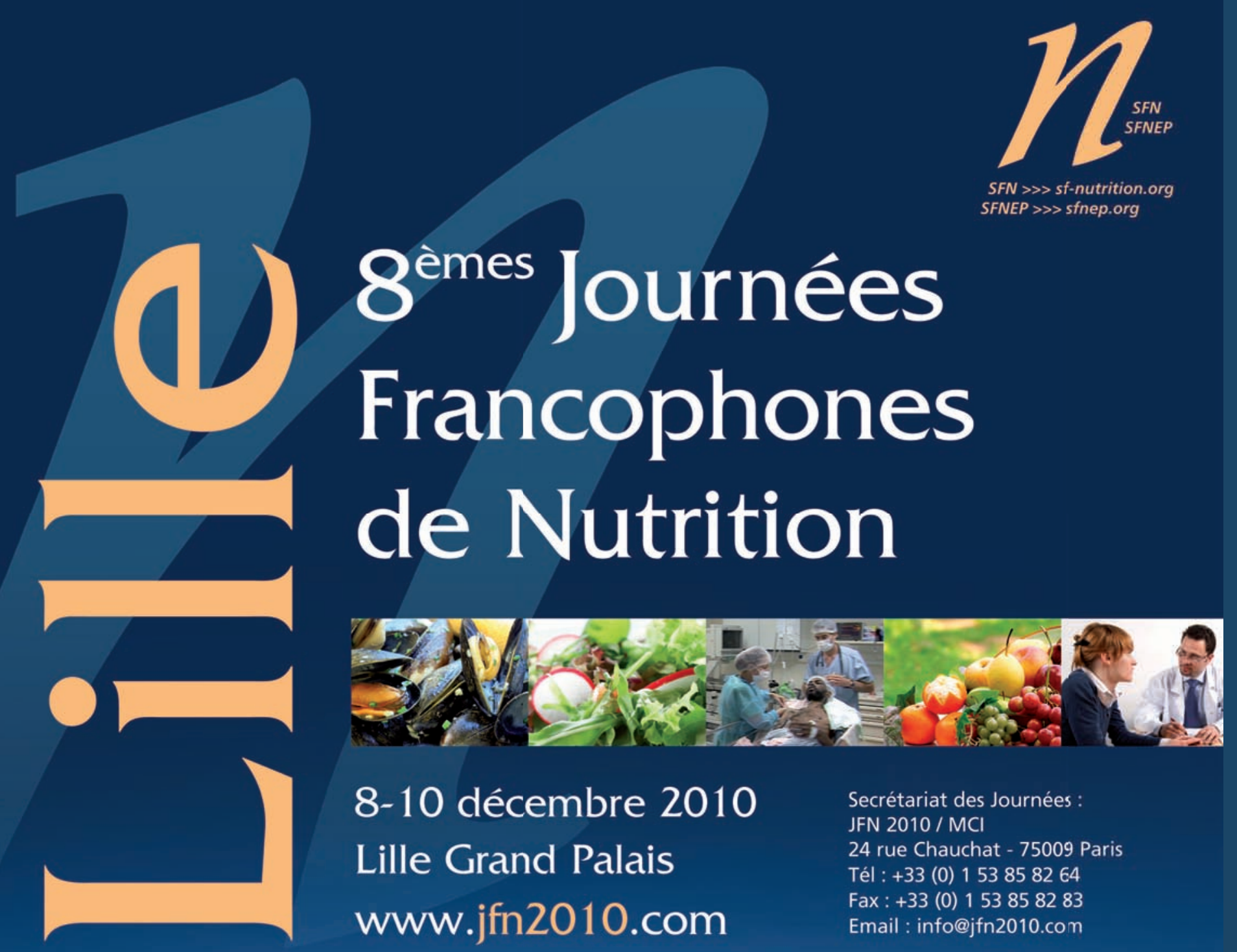

\section{8-10 décembre 2010 Lille Grand Palais} www.jfn2010.com
Secrétariat des Journées: JFN $2010 / \mathrm{MCl}$

24 rue Chauchat -75009 Paris

Tél : +33(0) 153858264

Fax : $+33(0) 153858283$

Email : info@jfn2010.con

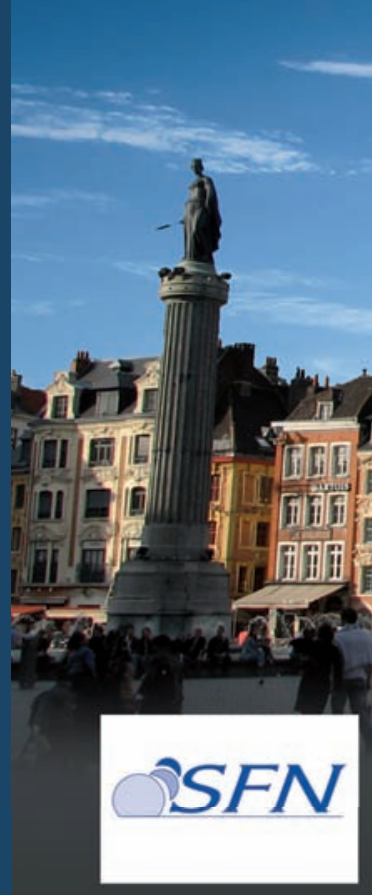

Société Française de Nutrition

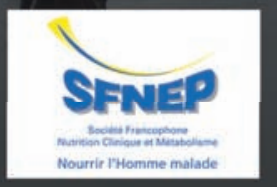

Société Francophone Nutrition Clinique et Métabolisme

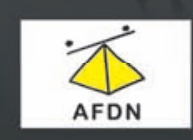

Association Française des Diététiciens Nutritionnistes

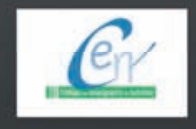

Collège des Enseignants de Nutrition

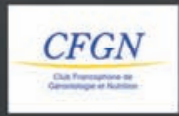

Club Francophone de Gérontologie et Nutrition

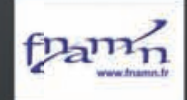

Féderation Nationale des Associations Medicales 